\title{
On block diagonal-Schur complements of the block strictly doubly diagonally dominant matrices
}

\section{Zhuo-Hong Huang*}

"Correspondence:
zhuohonghuang@cqut.edu.cn
School of Mathematics and
Statistics, Chongqing University of
Technology, Chongqing, 400054,
China

\begin{abstract}
As is well known, the diagonal-Schur complements of strictly diagonally dominant matrices are strictly diagonally dominant. In this paper, we verify the block diagonal-Schur complements of I-block strictly doubly diagonally dominant matrices are I-block strictly doubly diagonally dominant matrices, the same is true for II-block strictly doubly diagonally dominant matrices. The theoretical analysis is supported by numerical experiments.
\end{abstract}

Keywords: Schur complements; block diagonal-Schur complements; I-(II-) block strictly doubly diagonally dominant matrices; I-(II-) block strictly doubly diagonally dominant matrices; I-(II-) block H-matrix; comparison matrices; preconditioner

\section{Introduction}

The Schur complements and the diagonal-Schur complements have appeared to be useful tools in the study of matrix theory (see e.g., [1-3]), linear control theory (see e.g., [4]), numerical analysis (see e.g., [5-9]) and statistics (see e.g., [10, 11]), and so on. At the same time, given a matrix family, it is always interesting to see whether some important properties or structures of the family of matrices are inherited by the submatrices or by the matrices associated with the original matrices. These heritable properties have been used for the convergence of iterations in numerical analysis (see e.g., [12]).

A great deal of classic works on the relationships of the Schur complements and the diagonal-Schur complements with the original matrices have been contributed, for a complete survey of these works we refer to (see e.g., [12]). As is shown in [1, 2], the Schur complements of positive semidefinite matrices are positive semidefinite and the Schur complements of strictly diagonally dominant matrices are strictly diagonally dominant, the same is true for $M$-matrices, $H$-matrices, inverse $M$-matrices, strictly doubly diagonally dominant matrices and generalized strictly diagonally dominant. In addition, Liu and Huang [3] proposed that the diagonal-Schur complements of strictly diagonally dominant matrices are strictly diagonally dominant, the same is true for strictly $\gamma$-diagonally dominant matrices and strictly product $\gamma$-diagonally dominant matrices.

As regards the block matrix, the concept of a diagonally dominant matrix is extended and two kinds of block diagonally dominant matrices are proposed, i.e., I-block diagonally dominant matrices [13] and II-block diagonally dominant matrices [13]. Later, on the ba- 
sis of previous works, two kinds of generalized block strictly diagonally dominant matrices are also presented, in other words, I-block $H$-matrices [14] and II-block $H$-matrices [15]. Based on the above results, it is easy to see that a block diagonally dominant matrix is not always a diagonally dominant matrix; an example is given in [16], (2.6). Subsequently, Zhang et al. [17] showed that the Schur complement of I-(generalized) block diagonally dominant matrix is I-(generalized) block diagonally dominant, the same is true for II-(generalized) block diagonally dominant matrix.

Let $C^{n \times n}$ be the set of all $n \times n$ complex matrices. Suppose $A \in C^{n \times n}, N=\{1,2, \ldots, n\}$, and $|\alpha|$ equals the cardinality of $\alpha$. For nonempty index sets $\alpha, \beta \subseteq N$, we denote by $A(\alpha, \beta)$ the submatrix of $A \in C^{n \times n}$ lying in the rows indicated by $\alpha$ and the columns indicated by $\beta$. The submatrix $A(\alpha, \alpha)$ is abbreviated to $A(\alpha)$. Let $x^{T}$ be the transpose of the vector $x$ and $I_{n}$ be the $n \times n$ unit matrix.

Let $A=\left(a_{i j}\right) \in C^{n \times n}$. An $n \times n$ matrix $A$ is strictly diagonally dominant (abbreviated $\left.\mathrm{SD}_{n}\right)$, if

$$
\left|a_{i i}\right|>P_{i}(A), \quad P_{i}(A)=\sum_{j=1, j \neq i}\left|a_{i j}\right|, \quad i=1,2, \ldots, n .
$$

An $n \times n$ matrix $A$ is strictly generalized diagonally dominant (abbreviated $\mathrm{SGD}_{n}$ ), if there exists $D=\operatorname{diag}\left(d_{1}, \ldots, d_{n}\right)>0$, such that $A D$ is strictly diagonally dominant.

An $n \times n$ matrix $A$ is strictly doubly diagonally dominant (abbreviated $\mathrm{SDD}_{n}$ ), if

$$
\left|a_{i i} a_{j j}\right|>P_{i}(A) P_{j}(A), \quad \forall 1 \leq i<j \leq n .
$$

An $n \times n$ matrix $A$ is generalized strictly doubly diagonally dominant (abbreviated $\operatorname{SGDD}_{n}^{N_{1}, N_{2}}$ ), with $N_{1} \cup N_{2}=N, N_{1} \cap N_{2}=\emptyset$, and $\emptyset$ denoting the empty set, for all $i \in N_{1}$ and $j \in N_{2}$, if

$$
\left(\left|a_{i i}\right|-\gamma_{i}\right)\left(\left|a_{j j}\right|-\delta_{j}\right)>\gamma_{j} \delta_{i}
$$

where

$$
\gamma_{s}=\sum_{t \in N_{1}, t \neq s}\left|a_{s t}\right|, \quad \delta_{s}=\sum_{t \in N_{2}, t \neq s}\left|a_{s t}\right|, \quad \text { with } s=i \text { or } j
$$

Let $R^{n \times n}$ be the set of all $n \times n$ real matrices. For $A=\left(a_{i j}\right) \in R^{m \times n}$ and $B=\left(b_{i j}\right) \in R^{m \times n}$, we write $A \geq B$, if $a_{i j} \geq b_{i j}$ for all $i$, j. A real $n \times n$ matrix $A$ is called an $M$-matrix if $A=s I-B$, where $s \geq 0, B \geq 0$, and $s>\rho(B), \rho(B)$ is the spectral radius of $B$. Let $M_{n}$ denote the set of $n \times n M$-matrices.

Suppose $A \in C^{n \times n}$, the comparison matrix $\mu(A)=\left(\mu_{i j}\right)$, is defined by

$$
\mu_{i j}= \begin{cases}-\left|a_{i j}\right|, & i \neq j, \\ \left|a_{i j}\right|, & i=j .\end{cases}
$$

A complex $n \times n$ matrix $A$ is called an $H$-matrix if $\mu(A) \in M_{n}$. By $H_{n}$ is denoted the set of $n \times n H$-matrices.

In this paper, we propose the concept of the block diagonal-Schur complement on block matrices and study the properties on the diagonal-Schur complement of two kinds of (generalized) block doubly diagonally dominant matrices. 


\section{Definitions and lemmas}

Consider an $n \times n$ complex matrix $A$. Let $s(1 \leq s \leq n)$ be an arbitrary natural number and $A$ be partitioned into the following form:

$$
A=\left(\begin{array}{cccc}
A\left(\alpha_{1}, \alpha_{1}\right) & A\left(\alpha_{1}, \alpha_{2}\right) & \ldots & A\left(\alpha_{1}, \alpha_{s}\right) \\
A\left(\alpha_{2}, \alpha_{1}\right) & A\left(\alpha_{2}, \alpha_{2}\right) & \ldots & A\left(\alpha_{2}, \alpha_{s}\right) \\
\vdots & \vdots & \ddots & \vdots \\
A\left(\alpha_{s}, \alpha_{1}\right) & A\left(\alpha_{s}, \alpha_{2}\right) & \cdots & A\left(\alpha_{s}, \alpha_{s}\right)
\end{array}\right)
$$

where $\alpha_{0}=\emptyset$ and

$$
\alpha_{i}=\left\{\sum_{t=0}^{i-1}\left|\alpha_{t}\right|+1, \ldots, \sum_{t=0}^{i}\left|\alpha_{t}\right|\right\}, \quad i=1,2, \ldots, s, \sum_{t=0}^{s}\left|\alpha_{t}\right|=n
$$

with $A\left(\alpha_{t}, \alpha_{t}\right)$ being a $\left|\alpha_{t}\right| \times\left|\alpha_{t}\right|$ nonsingular principal submatrix of $A, t=1,2, \ldots, s$.

Let $C_{s}^{n \times n}$ be the set of all $s \times s$ block matrices in $C^{n \times n}$ partitioned as (1). Suppose $A=\left(A\left(\alpha_{l}, \alpha_{m}\right)\right)_{s \times s} \in C_{s}^{n \times n}$ and let $N(A)=\left(\left\|A\left(\alpha_{l}, \alpha_{m}\right)\right\|\right)_{s \times s}$ denote the norm matrix of block matrix $A$.

Let $\alpha \subset N, \alpha^{c}=N-\alpha$, and $A(\alpha)$ be nonsingular. The Schur complement of $A(\alpha)$ in $A$ is defined by

$$
A / A(\alpha)=A / \alpha=A\left(\alpha^{c}\right)-A\left(\alpha^{c}, \alpha\right)[A(\alpha)]^{-1} A\left(\alpha, \alpha^{c}\right),
$$

and the block diagonal-Schur complement of $A(\alpha)$ in $A$ is defined by

$$
A /{ }_{\circ} A(\alpha)=A / \circ \alpha=A\left(\alpha^{c}\right)-\left\{A\left(\alpha^{c}, \alpha\right)[A(\alpha)]^{-1} A\left(\alpha, \alpha^{c}\right)\right\} \circ E(\alpha),
$$

where 'o' denotes the Kronecker product symbol and

$$
\begin{aligned}
& \alpha=\alpha_{i_{1}} \cup \alpha_{i_{2}} \cup \cdots \cup \alpha_{i_{k}}, \quad \alpha^{c}=\alpha_{j_{1}} \cup \alpha_{j_{2}} \cup \cdots \cup \alpha_{j_{l}}, \\
& i_{1}<i_{2}<\cdots<i_{k}, j_{1}<j_{2}<\cdots<j_{l}, k+l=s, \\
& E_{|\alpha|}=\left(\begin{array}{cccc}
E_{\left|\alpha_{1}\right|} & & \\
& E_{\left|\alpha_{2}\right|} & \\
& & & \\
& & & \\
& & \left|\alpha_{s}\right|
\end{array}\right), \quad E_{\left|\alpha_{i}\right|}=\left(\begin{array}{cccc}
1 & 1 & \ldots & 1 \\
1 & 1 & \ldots & 1 \\
\vdots & \vdots & \ddots & \vdots \\
1 & 1 & \ldots & 1
\end{array}\right)_{\left|\alpha_{i}\right| \times\left|\alpha_{i}\right|},
\end{aligned}
$$

with $i=1,2, \ldots, s$.

Definition 2.1 [13] Let $A=\left(A\left(\alpha_{l}, \alpha_{m}\right)\right)_{s \times s} \in C_{s}^{n \times n}$ and $A\left(\alpha_{l}, \alpha_{l}\right)(l=1,2, \ldots, s)$ be nonsingular. If

$$
\left\|\left[A\left(\alpha_{l}, \alpha_{l}\right)\right]^{-1}\right\|^{-1}>\sum_{\substack{m=1 \\ m \neq l}}^{s}\left\|A\left(\alpha_{l}, \alpha_{m}\right)\right\|, \quad \forall l \in s,
$$

then $A$ is an I-block strictly diagonally dominant matrix (abbreviated $\left.\mathrm{I}-\mathrm{BSD}_{s}\right)$. 
Definition 2.2 [13] Let $A=\left(A\left(\alpha_{l}, \alpha_{m}\right)\right)_{s \times s} \in C_{s}^{n \times n}$ and $A\left(\alpha_{l}, \alpha_{l}\right)(l=1,2, \ldots, s)$ be nonsingular. If

$$
\sum_{\substack{m=1 \\ m \neq l}}^{s}\left\|\left[A\left(\alpha_{l}, \alpha_{l}\right)\right]^{-1} A\left(\alpha_{l}, \alpha_{m}\right)\right\|<1, \quad \forall l \in s
$$

then $A$ is an II-block strictly diagonally dominant matrix (abbreviated II-BSD ${ }_{s}$ ).

Definition 2.3 [5] Let $A=\left(A\left(\alpha_{l}, \alpha_{m}\right)\right)_{s \times s} \in C_{s}^{n \times n}$ and $A\left(\alpha_{l}, \alpha_{l}\right)(l=1,2, \ldots, s)$ be nonsingular. For $1 \leq i<j \leq s$, if and only if

$$
\left\|\left[A\left(\alpha_{i}, \alpha_{i}\right)\right]^{-1}\right\|^{-1}\left\|\left[A\left(\alpha_{j}, \alpha_{j}\right)\right]^{-1}\right\|^{-1}>\sum_{\substack{m=1 \\ m \neq i}}^{s}\left\|A\left(\alpha_{i}, \alpha_{m}\right)\right\| \sum_{\substack{m=1 \\ m \neq j}}^{s}\left\|A\left(\alpha_{j}, \alpha_{m}\right)\right\|,
$$

then $A$ is an I-block strictly doubly diagonally dominant matrix (abbreviated I-BSDD ${ }_{s}$ ).

Definition 2.4 [5] Let $A=\left(A\left(\alpha_{l}, \alpha_{m}\right)\right)_{s \times s} \in C_{s}^{n \times n}$ and $A\left(\alpha_{l}, \alpha_{l}\right)(l=1,2, \ldots, s)$ be nonsingular. For $1 \leq i<j \leq s$, if and only if

$$
\sum_{\substack{m=1 \\ m \neq i}}^{s}\left\|\left[A\left(\alpha_{i}, \alpha_{i}\right)\right]^{-1} A\left(\alpha_{i}, \alpha_{m}\right)\right\| \sum_{\substack{m=1 \\ m \neq j}}^{s}\left\|\left[A\left(\alpha_{j}, \alpha_{j}\right)\right]^{-1} A\left(\alpha_{j}, \alpha_{m}\right)\right\|<1,
$$

then $A$ is an II-block strictly doubly diagonally dominant matrix (abbreviated II-BSDD ${ }_{s}$ ).

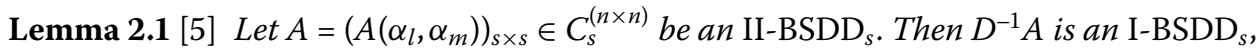
where $D=\operatorname{diag}\left(A\left(\alpha_{1}, \alpha_{1}\right), A\left(\alpha_{2}, \alpha_{2}\right), \ldots, A\left(\alpha_{s}, \alpha_{s}\right)\right)$.

Remark 2.1 [5] If $A$ is an $\mathrm{I}_{-\mathrm{BSD}}$ (or $\mathrm{I}-\mathrm{BSDD}_{s}$ ), according to the following inequality:

$$
\left\|A\left(\alpha_{l}, \alpha_{l}\right) A\left(\alpha_{l}, \alpha_{m}\right)\right\| \leq\left\|A\left(\alpha_{l}, \alpha_{l}\right)\right\|\left\|A\left(\alpha_{l}, \alpha_{m}\right)\right\|,
$$

then $A$ is an $\mathrm{II}-\mathrm{BSD}_{s}($ or II-BSDD $)$.

Definition $2.5[14,15]$ Let $A=\left(A\left(\alpha_{l}, \alpha_{m}\right)\right)_{s \times s} \in C_{s}^{n \times n}$ and $A\left(\alpha_{l}, \alpha_{l}\right)(l=1,2, \ldots, s)$ be nonsingular. If the comparison matrices of block matrix $A, \mu_{I}(A)=\left(\omega_{l, m}\right) \in R^{s \times s}$ or $\mu_{I I}(A)=$ $\left(\varpi_{l, m}\right) \in R^{s \times s}$ is an $M$-matrix, respectively, where

$$
\begin{aligned}
& \omega_{l m}= \begin{cases}\left\|\left[A\left(\alpha_{l}, \alpha_{l}\right)\right]^{-1}\right\|^{-1}, & \text { if } l=m, \\
-\left\|A\left(\alpha_{l}, \alpha_{m}\right)\right\|, & \text { if } l \neq m,\end{cases} \\
& \varpi_{l m}= \begin{cases}1, & \text { if } l=m, \\
-\left\|\left[A\left(\alpha_{l}, \alpha_{l}\right)\right]^{-1} A\left(\alpha_{l}, \alpha_{m}\right)\right\|, & \text { if } l \neq m,\end{cases}
\end{aligned}
$$

then $A$ is called an I-block $H$-matrix or an II-block $H$-matrix.

Lemma 2.2 [13] Let $A=\left(A\left(\alpha_{l}, \alpha_{m}\right)\right)_{s \times s} \in \mathrm{I}$-(II-) block $H$-matrix. Then $A$ is nonsingular. 
Remark 2.2 If $A$ is an I-(II-)BSD ${ }_{s}$ or an I-(II-)BSDD ${ }_{s}$, by (4)-(7), then $\mu_{I}(A)\left(\right.$ or $\left.\mu_{I I}(A)\right)$ is an $M$-matrix. Further, by Definition 2.5 and Lemma 2.2, then $A$ is a nonsingular I-(II-) block $H$-matrix.

Lemma 2.3 [15] If $A \in \mathrm{SD}_{n}, \mathrm{SDD}_{n}, \mathrm{SGD}_{n}$ or $\mathrm{SGDD}_{n}^{N_{1}, N_{2}}$. Then $\mu(A)$ is an M-matrix, i.e., $A$ is an H-matrix.

Lemma 2.4 [1] Let $A \in C^{n \times n}$. If $\|A\|<1$, then $I_{n}-A$ is nonsingular and

$$
\left\|\left(I_{n}-A\right)^{-1}\right\| \leq \frac{1}{1-\|A\|},
$$

where $I_{n}$ denotes the $n \times n$ identity matrix.

Lemma $2.5[18]$ Let $A=\left(A\left(\alpha_{l}, \alpha_{m}\right)\right)_{s \times s} \in \mathrm{I}-(\mathrm{II}-) \mathrm{BSD}_{s}$. For all $t=1,2, \ldots, l$, then

$$
\psi_{t}=1-\left\|\left[A\left(\alpha_{j_{t}}, \alpha_{j_{t}}\right)\right]^{-1}\left(A\left(\alpha_{j_{t}}, \alpha_{i_{1}}\right), \ldots, A\left(\alpha_{j_{t}}, \alpha_{i_{k}}\right)\right)[A(\alpha)]^{-1}\left(\begin{array}{c}
A\left(\alpha_{i_{1}}, \alpha_{j_{t}}\right) \\
\vdots \\
A\left(\alpha_{i_{k}}, \alpha_{j_{t}}\right)
\end{array}\right)\right\|>0 .
$$

\section{On block diagonal-Schur complement of I-(II-)BSDD}

In this section, to verify the heritable properties of the block diagonal-Schur complements from the original matrix I-BSDD $s$ and $\mathrm{I}_{-} \mathrm{BSDD}_{s}$, we only need to consider two cases as follows:

(1) If $A$ is an I-BSDD but is not an I-BSD , by Definition 2.3, there exists one and only one index $i_{0}, A\left(\alpha_{i_{0}}, \alpha_{i_{0}}\right)$ being nonsingular and such that

$$
\left\|\left[A\left(\alpha_{i_{0}}, \alpha_{i_{0}}\right)\right]^{-1}\right\|^{-1} \leq P_{i_{0}}(A) .
$$

(2) If $A$ is an II-BSDD but not an II-BSD , by Definition 2.4, there exists one and only one index $i_{0}, A\left(\alpha_{i_{0}}, \alpha_{i_{0}}\right)$ being nonsingular and satisfying

$$
\sum_{r=1}^{s}\left\|\left[A\left(\alpha_{i_{0}}, \alpha_{i_{0}}\right)\right]^{-1} A\left(\alpha_{i_{0}}, \alpha_{r}\right)\right\| \geq 1
$$

Theorem 3.1 Let $A$ be an $n \times n \mathrm{I}_{-} \mathrm{BSDD}_{s}$ but be not an $n \times n \mathrm{I}_{-} \mathrm{BSD}_{s}$, and $i_{0}\left(1 \leq i_{0} \leq s\right)$ satisfy the condition in (9). For any index set $\alpha \subseteq N$, writing $\alpha=\alpha_{i_{1}} \cup \alpha_{i_{2}} \cup \cdots \cup \alpha_{i_{k}}$ and $\alpha^{c}=\alpha_{j_{1}} \cup \alpha_{j_{2}} \cup \cdots \cup \alpha_{j_{l}}$, with $k+l=s$, then:

(i) If $\alpha_{i_{0}} \subseteq \alpha$, then $A /{ }_{\circ} \alpha \in \mathrm{I}_{-} \mathrm{BSD}_{l}$.

(ii) If $\alpha_{i_{0}} \subseteq \alpha^{c}$, then $A /{ }_{\circ} \alpha \in \mathrm{I}_{-} \mathrm{BSDD}_{l}$.

Proof Without loss of generality, we can assume $A /{ }_{\circ} \alpha=\left(\tilde{A}\left(\alpha_{t}, \alpha_{r}\right)\right)$ and denote

$$
\begin{aligned}
& \Phi_{\omega}=\left(A\left(\alpha_{j_{\omega}}, \alpha_{i_{1}}\right), \ldots, A\left(\alpha_{j_{\omega}}, \alpha_{i_{k}}\right)\right)[A(\alpha)]^{-1}\left(\begin{array}{c}
A\left(\alpha_{i_{1}}, \alpha_{j_{\omega}}\right) \\
\vdots \\
A\left(\alpha_{i_{k}}, \alpha_{j_{\omega}}\right)
\end{array}\right), \\
& K_{\omega}=\left(\left\|A\left(\alpha_{j_{\omega}}, \alpha_{i_{1}}\right)\right\|, \ldots,\left\|A\left(\alpha_{j_{\omega}}, \alpha_{i_{k}}\right)\right\|\right), \quad H_{v}=\left(\left\|A\left(\alpha_{i_{1}}, \alpha_{j_{v}}\right)\right\|, \ldots,\left\|A\left(\alpha_{i_{k}}, \alpha_{j_{v}}\right)\right\|\right)^{T}, \\
& \Psi_{\omega}=K_{\omega} \cdot \mu_{I}\left\{[A(\alpha)]^{-1}\right\} \cdot H_{\omega}, \quad \Upsilon_{\omega, v}=K_{\omega} \cdot\left\{\mu_{I}[A(\alpha)]\right\}^{-1} \cdot H_{v}, \quad \text { with } \omega, v=t, u .
\end{aligned}
$$


By the definition of the block diagonal-Schur complement (3), denote $\left|\alpha_{j_{t}}\right|=J_{t}$. According to Remark 2.2, we obtain $\left\|\left[A\left(\alpha_{j_{\omega}}, \alpha_{j_{\omega}}\right)\right]^{-1} \Phi_{\omega}\right\|<1, \omega=t, u$. Further, consider the following two cases:

(i) If $\alpha_{i_{0}} \subseteq \alpha$, then

$$
\begin{aligned}
& \left\|\left[\tilde{A}\left(\alpha_{t}, \alpha_{t}\right)\right]^{-1}\right\|^{-1}-\sum_{\substack{r=1 \\
r \neq t}}^{l}\left\|\tilde{A}\left(\alpha_{t}, \alpha_{r}\right)\right\| \\
& =\left\|\left\{A\left(\alpha_{j_{t}}, \alpha_{j_{t}}\right)-\Phi_{t}\right\}^{-1}\right\|^{-1}-\sum_{\substack{r=1 \\
r \neq t}}^{l}\left\|A\left(\alpha_{j_{t}}, \alpha_{j_{r}}\right)\right\| \\
& =\left\|\left\{I_{J_{t}}-\left[A\left(\alpha_{j_{t}}, \alpha_{j_{t}}\right)\right]^{-1} \Phi_{t}\right\}^{-1}\left[A\left(\alpha_{j_{t}}, \alpha_{j_{t}}\right)\right]^{-1}\right\|^{-1}-\sum_{\substack{r=1 \\
r \neq t}}^{l}\left\|A\left(\alpha_{j_{t}}, \alpha_{j_{r}}\right)\right\| \\
& \geq\left\|\left\{I_{j_{t}}-\left[A\left(\alpha_{j_{t}}, \alpha_{j_{t}}\right)\right]^{-1} \Phi_{t}\right\}^{-1}\right\|^{-1}\left\|\left[A\left(\alpha_{j_{t}}, \alpha_{j_{t}}\right)\right]^{-1}\right\|^{-1}-\sum_{\substack{r=1 \\
r \neq t}}^{l}\left\|A\left(\alpha_{j_{t}}, \alpha_{j_{r}}\right)\right\| \\
& \geq\left\{1-\left\|\left[A\left(\alpha_{j_{t}}, \alpha_{j_{t}}\right)\right]^{-1} \Phi_{t}\right\|\right\}\left\|\left[A\left(\alpha_{j_{t}}, \alpha_{j_{t}}\right)\right]^{-1}\right\|^{-1}-\sum_{\substack{r=1 \\
r \neq t}}^{l}\left\|A\left(\alpha_{j_{t}}, \alpha_{j_{r}}\right)\right\| \\
& =\left\|\left[A\left(\alpha_{j_{t}}, \alpha_{j_{t}}\right)\right]^{-1}\right\|^{-1}-\left\|\left[A\left(\alpha_{j_{t}}, \alpha_{j_{t}}\right)\right]^{-1}\right\|^{-1}\left\|\left[A\left(\alpha_{j_{t}}, \alpha_{j_{t}}\right)\right]^{-1} \Phi_{t}\right\|-\sum_{\substack{r=1 \\
r \neq t}}^{l}\left\|A\left(\alpha_{j_{t}}, \alpha_{j_{r}}\right)\right\| \\
& \geq\left\|\left[A\left(\alpha_{j_{t}}, \alpha_{j_{t}}\right)\right]^{-1}\right\|^{-1}-\left\|\left[A\left(\alpha_{j_{t}}, \alpha_{j_{t}}\right)\right]^{-1}\right\|^{-1}\left\|\left[A\left(\alpha_{j_{t}}, \alpha_{j_{t}}\right)\right]^{-1}\right\|\left\|\Phi_{t}\right\|-\sum_{\substack{r=1 \\
r \neq t}}^{l}\left\|A\left(\alpha_{j_{t}}, \alpha_{j_{r}}\right)\right\| \\
& \geq\left\|\left[A\left(\alpha_{j_{t}}, \alpha_{j_{t}}\right)\right]^{-1}\right\|^{-1}-\sum_{\substack{r=1 \\
r \neq t}}^{l}\left\|A\left(\alpha_{j_{t}}, \alpha_{j_{r}}\right)\right\|-\Psi_{\omega} \\
& \geq\left\|\left[A\left(\alpha_{j_{t}}, \alpha_{j_{t}}\right)\right]^{-1}\right\|^{-1}-\sum_{\substack{r=1 \\
r \neq t}}^{l}\left\|A\left(\alpha_{j_{t}}, \alpha_{j_{r}}\right)\right\|-\Upsilon_{\omega, \omega} \\
& \triangleq \frac{\operatorname{det} B_{1}}{\operatorname{det}\left[\mu_{I}(A(\alpha))\right]}
\end{aligned}
$$

where

$$
B_{1}=\left(\begin{array}{cc}
\left\|\left[A\left(\alpha_{j_{t}}, \alpha_{j_{t}}\right)\right]^{-1}\right\|^{-1}-\sum_{\substack{r=1 \\
r \neq t}}^{l}\left\|A\left(\alpha_{j_{t}}, \alpha_{j_{r}}\right)\right\| & -K_{t} \\
-H_{t} & \mu_{I}[A(\alpha)]
\end{array}\right) .
$$

Since $A$ is an I-BSDD ${ }_{s}, \alpha_{i_{0}} \subseteq \alpha$, and $\forall \alpha_{j_{t}} \subseteq \alpha^{c}$,

$$
\left\|\left[A\left(\alpha_{j_{t}}, \alpha_{j_{t}}\right)\right]^{-1}\right\|^{-1}-\sum_{\substack{r=1 \\ r \neq t}}^{l}\left\|A\left(\alpha_{j_{t}}, \alpha_{j_{r}}\right)\right\|>\sum_{r=1}^{k}\left\|A\left(\alpha_{j_{t}}, \alpha_{i_{r}}\right)\right\| .
$$


For $\forall \alpha_{i_{x}} \subseteq \alpha, x=1,2, \ldots, k$, if $i_{x} \neq i_{0}$, then

$$
\left\|\left[A\left(\alpha_{i_{x}}, \alpha_{i_{x}}\right)\right]^{-1}\right\|^{-1}>\sum_{\substack{r=1 \\ r \neq i_{x}}}^{s}\left\|A\left(\alpha_{i_{x}}, \alpha_{r}\right)\right\| \geq \sum_{\substack{r=1 \\ r \neq x}}^{k}\left\|A\left(\alpha_{i_{x}}, \alpha_{i_{r}}\right)\right\|+\left\|A\left(\alpha_{i_{x}}, \alpha_{j_{t}}\right)\right\|,
$$

if $i_{x}=i_{0}$, by Definition 2.3 and the inequality (9), we have

$$
\begin{aligned}
& \left\{\left\|\left[A\left(\alpha_{j_{t}}, \alpha_{j_{t}}\right)\right]^{-1}\right\|^{-1}-\sum_{\substack{r=1 \\
r \neq t}}^{l}\left\|A\left(\alpha_{j_{t}}, \alpha_{j_{r}}\right)\right\|\right\}\left\|\left[A\left(\alpha_{i_{0}}, \alpha_{i_{0}}\right)\right]^{-1}\right\|^{-1} \\
& =\left\|\left[A\left(\alpha_{j_{t}}, \alpha_{j_{t}}\right)\right]^{-1}\right\|^{-1}\left\|\left[A\left(\alpha_{i_{0}}, \alpha_{i_{0}}\right)\right]^{-1}\right\|^{-1}-\left\|\left[A\left(\alpha_{i_{0}}, \alpha_{i_{0}}\right)\right]^{-1}\right\|^{-1} \sum_{\substack{r=1 \\
r \neq t}}^{l}\left\|A\left(\alpha_{j_{t}}, \alpha_{j_{r}}\right)\right\| \\
& >\sum_{\substack{r=1 \\
r \neq j_{t}}}^{s}\left\|A\left(\alpha_{j_{t}}, \alpha_{r}\right)\right\| \sum_{\substack{r=1 \\
r \neq i_{0}}}^{s}\left\|A\left(\alpha_{i_{0}}, \alpha_{r}\right)\right\|-\sum_{\substack{r=1 \\
r \neq i_{0}}}^{s}\left\|A\left(\alpha_{i_{0}}, \alpha_{r}\right)\right\| \sum_{\substack{r=1 \\
r \neq t}}^{l}\left\|A\left(\alpha_{j_{t}}, \alpha_{j_{r}}\right)\right\| \\
& =\left\{\sum_{\substack{r=1 \\
r \neq j_{t}}}^{s}\left\|A\left(\alpha_{j_{t}}, \alpha_{r}\right)\right\|-\sum_{\substack{r=1 \\
r \neq t}}^{l}\left\|A\left(\alpha_{j_{t}}, \alpha_{j_{r}}\right)\right\|\right\} \sum_{\substack{r=1 \\
r \neq i_{0}}}^{s}\left\|A\left(\alpha_{i_{0}}, \alpha_{r}\right)\right\| \\
& \geq \sum_{r=1}^{k}\left\|A\left(\alpha_{j_{t}}, \alpha_{i_{r}}\right)\right\|\left\{\sum_{\substack{r=1 \\
i_{r} \neq i_{0}}}^{k}\left\|A\left(\alpha_{i_{0}}, \alpha_{i_{r}}\right)\right\|+\left\|A\left(\alpha_{i_{0}}, \alpha_{j_{t}}\right)\right\|\right\} .
\end{aligned}
$$

Thus, $B_{1} \in \mathrm{SDD}_{k+1}$. Further, by Lemma 2.3, we have $B_{1}=\mu\left(B_{1}\right) \in M_{k+1}$ and $\mu_{I}[A(\alpha)] \in$ $M_{k}$. Therefore, $\operatorname{det}\left(B_{1}\right)>0$ and $\operatorname{det}\left[\mu_{I}(A(\alpha))\right]>0$, i.e.,

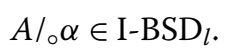

(ii) If $\alpha_{i_{0}} \subseteq \alpha^{c}$, for $\forall t, u=1,2, \ldots, l$ and $t \neq u$, we have

$$
\begin{aligned}
& \left\|\left[\tilde{A}\left(\alpha_{t}, \alpha_{t}\right)\right]^{-1}\right\|^{-1}\left\|\left[\tilde{A}\left(\alpha_{u}, \alpha_{u}\right)\right]^{-1}\right\|^{-1}-\sum_{\substack{r=1 \\
r \neq t}}^{l}\left\|\tilde{A}\left(\alpha_{t}, \alpha_{r}\right)\right\| \sum_{\substack{r=1 \\
r \neq u}}^{l}\left\|\tilde{A}\left(\alpha_{u}, \alpha_{r}\right)\right\| \\
& =\left\|\left\{A\left(\alpha_{j_{t}}, \alpha_{j_{t}}\right)-\Phi_{t}\right\}^{-1}\right\|^{-1}\left\|\left\{A\left(\alpha_{j_{u}}, \alpha_{j_{u}}\right)-\Phi_{u}\right\}^{-1}\right\|^{-1} \\
& \quad-\sum_{\substack{r=1 \\
r \neq t}}^{l}\left\|A\left(\alpha_{j_{t}}, \alpha_{j_{r}}\right)\right\| \sum_{\substack{r=1 \\
r \neq u}}^{l}\left\|A\left(\alpha_{j_{u}}, \alpha_{j_{r}}\right)\right\| \\
& =\left\|\left\{I_{J_{t}}-\left[A\left(\alpha_{j_{t}}, \alpha_{j_{t}}\right)\right]^{-1} \Phi_{t}\right\}^{-1}\left[A\left(\alpha_{j_{t}}, \alpha_{j_{t}}\right)\right]^{-1}\right\|^{-1} \\
& \quad \times\left\|\left\{I_{J_{u}}-\left[A\left(\alpha_{j_{u}}, \alpha_{j_{u}}\right)\right]^{-1} \Phi_{u}\right\}^{-1}\left[A\left(\alpha_{j_{u}}, \alpha_{j_{u}}\right)\right]^{-1}\right\|^{-1} \\
& \quad-\sum_{\substack{r=1 \\
r \neq t}}^{l}\left\|A\left(\alpha_{j_{t}}, \alpha_{j_{r}}\right)\right\| \sum_{\substack{r=1 \\
r \neq u}}^{l}\left\|A\left(\alpha_{j_{u}}, \alpha_{j_{r}}\right)\right\| \\
& \geq\left\|\left\{I_{J_{t}}-\left[A\left(\alpha_{j_{t}}, \alpha_{j_{t}}\right)\right]^{-1} \Phi_{t}\right\}^{-1}\right\|^{-1}\left\|\left\{I_{J_{u}}-\left[A\left(\alpha_{j_{u}}, \alpha_{j_{u}}\right)\right]^{-1} \Phi_{u}\right\}^{-1}\right\|^{-1}
\end{aligned}
$$




$$
\begin{aligned}
& \times\left\|\left[A\left(\alpha_{j_{t}}, \alpha_{j_{t}}\right)\right]^{-1}\right\|^{-1}\left\|\left[A\left(\alpha_{j_{u}}, \alpha_{j_{u}}\right)\right]^{-1}\right\|^{-1}-\sum_{\substack{r=1 \\
r \neq t}}^{l}\left\|A\left(\alpha_{j_{t}}, \alpha_{j_{r}}\right)\right\| \sum_{\substack{r=1 \\
r \neq u}}^{l}\left\|A\left(\alpha_{j_{u}}, \alpha_{j_{r}}\right)\right\| \\
& \geq\left\{1-\left\|\left[A\left(\alpha_{j_{t}}, \alpha_{j_{t}}\right)\right]^{-1} \Phi_{t}\right\|\right\}\left\{1-\left\|\left[A\left(\alpha_{j_{u}}, \alpha_{j_{u}}\right)\right]^{-1} \Phi_{u}\right\|\right\} \\
& \times\left\|\left[A\left(\alpha_{j_{t}}, \alpha_{j_{t}}\right)\right]^{-1}\right\|^{-1}\left\|\left[A\left(\alpha_{j_{u}}, \alpha_{j_{u}}\right)\right]^{-1}\right\|^{-1}-\sum_{\substack{r=1 \\
r \neq t}}^{l}\left\|A\left(\alpha_{j_{t}}, \alpha_{j_{r}}\right)\right\| \sum_{\substack{r=1 \\
r \neq u}}^{l}\left\|A\left(\alpha_{j_{u}}, \alpha_{j_{r}}\right)\right\| \\
& \geq\left\{1-\left\|\left[A\left(\alpha_{j_{t}}, \alpha_{j_{t}}\right)\right]^{-1}\right\|\left\|\Phi_{t}\right\|\right\}\left\{1-\left\|\left[A\left(\alpha_{j_{u}}, \alpha_{j_{u}}\right)\right]^{-1}\right\|\left\|\Phi_{u}\right\|\right\} \\
& \times\left\|\left[A\left(\alpha_{j_{t}}, \alpha_{j_{t}}\right)\right]^{-1}\right\|^{-1}\left\|\left[A\left(\alpha_{j_{u}}, \alpha_{j_{u}}\right)\right]^{-1}\right\|^{-1}-\sum_{\substack{r=1 \\
r \neq t}}^{l}\left\|A\left(\alpha_{j_{t}}, \alpha_{j_{r}}\right)\right\| \sum_{\substack{r=1 \\
r \neq u}}^{l}\left\|A\left(\alpha_{j_{u}}, \alpha_{j_{r}}\right)\right\| \\
& =\left\{\left\|\left[A\left(\alpha_{j_{t}}, \alpha_{j_{t}}\right)\right]^{-1}\right\|^{-1}-\left\|\Phi_{t}\right\|\right\}\left\{\left\|\left[A\left(\alpha_{j_{u}}, \alpha_{j_{u}}\right)\right]^{-1}\right\|^{-1}-\left\|\Phi_{u}\right\|\right\} \\
& -\sum_{\substack{r=1 \\
r \neq t}}^{l}\left\|A\left(\alpha_{j_{t}}, \alpha_{j_{r}}\right)\right\| \sum_{\substack{r=1 \\
r \neq u}}^{l}\left\|A\left(\alpha_{j_{u}}, \alpha_{j_{r}}\right)\right\| \\
& \geq\left\{\left\|\left[A\left(\alpha_{j_{t}}, \alpha_{j_{t}}\right)\right]^{-1}\right\|^{-1}-\Psi_{t}\right\}\left\{\left\|\left[A\left(\alpha_{j_{u}}, \alpha_{j_{u}}\right)\right]^{-1}\right\|^{-1}-\Psi_{u}\right\} \\
& -\sum_{\substack{r=1 \\
r \neq t}}^{l}\left\|A\left(\alpha_{j_{t}}, \alpha_{j_{r}}\right)\right\| \sum_{\substack{r=1 \\
r \neq u}}^{l}\left\|A\left(\alpha_{j_{u}}, \alpha_{j_{r}}\right)\right\| \\
& \geq\left\{\left\|\left[A\left(\alpha_{j_{t}}, \alpha_{j_{t}}\right)\right]^{-1}\right\|^{-1}-\Upsilon_{t t}\right\}\left\{\left\|\left[A\left(\alpha_{j_{u}}, \alpha_{j_{u}}\right)\right]^{-1}\right\|^{-1}-\Upsilon_{u u}\right\} \\
& -\sum_{\substack{r=1 \\
r \neq t}}^{l}\left\|A\left(\alpha_{j_{t}}, \alpha_{j_{r}}\right)\right\| \sum_{\substack{r=1 \\
r \neq u}}^{l}\left\|A\left(\alpha_{j_{u}}, \alpha_{j_{r}}\right)\right\| \\
& \geq\left\{\left\|\left[A\left(\alpha_{j_{t}}, \alpha_{j_{t}}\right)\right]^{-1}\right\|^{-1}-\Upsilon_{t t}\right\}\left\{\left\|\left[A\left(\alpha_{j_{u}}, \alpha_{j_{u}}\right)\right]^{-1}\right\|^{-1}-\Upsilon_{u u}\right\} \\
& -\left\{\sum_{r=1}^{l}\left\|A\left(\alpha_{j_{t}}, \alpha_{j_{r}}\right)\right\|+\Upsilon_{t u}\right\}\left\{\sum_{r=1}^{l}\left\|A\left(\alpha_{j_{u}}, \alpha_{j_{r}}\right)\right\|+\Upsilon_{u t}\right\} \\
& \triangleq \frac{\operatorname{det} B_{2}}{\operatorname{det}\left[\mu_{I}(A)(\alpha)\right]},
\end{aligned}
$$

where

$$
B_{2}=\left(\begin{array}{ccc}
\left\|\left[A\left(\alpha_{j_{t}}, \alpha_{j_{t}}\right)\right]^{-1}\right\|^{-1} & -\sum_{\substack{r=1 \\
r \neq t}}^{l}\left\|A\left(\alpha_{j_{t}}, \alpha_{j_{r}}\right)\right\| & -K_{t} \\
-\sum_{\substack{r=1 \\
r \neq u}}^{l}\left\|A\left(\alpha_{j_{u}}, \alpha_{j_{r}}\right)\right\| & \left\|\left[A\left(\alpha_{j_{u}}, \alpha_{j_{u}}\right)\right]^{-1}\right\|^{-1} & -K_{u} \\
-H_{t} & -H_{u} & \mu_{I}(A)(\alpha)
\end{array}\right) .
$$

Since $A$ is an $\mathrm{I}-\mathrm{BSDD}_{s}, \alpha_{i_{0}} \subseteq \alpha^{c}$ and $\alpha_{j_{\omega}} \subseteq \alpha^{c}, \omega=t, u$,

$$
\left\|\left[A\left(\alpha_{j_{t}}, \alpha_{j_{t}}\right)\right]^{-1}\right\|^{-1}\left\|\left[A\left(\alpha_{j_{u}}, \alpha_{j_{u}}\right)\right]^{-1}\right\|^{-1}>\sum_{\substack{r=1 \\ r \neq j t}}^{s}\left\|A\left(\alpha_{j_{t}}, \alpha_{r}\right)\right\| \sum_{\substack{r=1 \\ r \neq j_{u}}}^{s}\left\|A\left(\alpha_{j_{u}}, \alpha_{r}\right)\right\| .
$$


For $\forall \alpha_{i_{x}} \subseteq \alpha, x=1,2, \ldots, k$, and $\alpha_{j_{w}} \subseteq \alpha^{c}, w=t, u$,

$$
\begin{aligned}
& \left\|\left[A\left(\alpha_{i_{x}}, \alpha_{i_{x}}\right)\right]^{-1}\right\|^{-1}\left\|\left[A\left(\alpha_{j_{\omega}}, \alpha_{j_{\omega}}\right)\right]^{-1}\right\|^{-1} \\
& \quad>\left\{\sum_{\substack{r=1 \\
r \neq x}}^{k}\left\|A\left(\alpha_{i_{x}}, \alpha_{i_{r}}\right)\right\|+\left\|A\left(\alpha_{i_{x}}, \alpha_{j_{t}}\right)\right\|+\left\|A\left(\alpha_{i_{x}}, \alpha_{j_{u}}\right)\right\|\right\} \sum_{\substack{r=1 \\
r \neq j_{\omega}}}^{s}\left\|A\left(\alpha_{j_{\omega}}, \alpha_{r}\right)\right\|
\end{aligned}
$$

and

$$
\left\|\left[A\left(\alpha_{i_{x}}, \alpha_{i_{x}}\right)\right]^{-1}\right\|^{-1}>\left\{\sum_{\substack{r=1 \\ r \neq x}}^{k}\left\|A\left(\alpha_{i_{x}}, \alpha_{i_{r}}\right)\right\|+\left\|A\left(\alpha_{i_{x}}, \alpha_{j_{u}}\right)\right\|+\left\|A\left(\alpha_{i_{x}}, \alpha_{j_{t}}\right)\right\|\right\} .
$$

Thus, $B_{2} \in \mathrm{SDD}_{k+2}$. Further, by Lemma 2.3, we have $B_{2}=\mu\left(B_{2}\right) \in M_{k+2}$ and $\mu_{I}[A(\alpha)] \in$ $M_{k}$. Therefore, $\operatorname{det}\left(B_{2}\right)>0$ and $\operatorname{det}\left[\mu_{I}(A)(\alpha)\right]>0$, i.e.,

$A /{ }_{\circ} \alpha \in \mathrm{I}-\mathrm{BSDD}_{l}$.

Combining the proof of (i) and (ii), we complete the proof of Theorem 3.1.

Theorem 3.2 Let $A$ be an $n \times n$ II-BSDD but be not an $n \times n$ II-BSD , and $i_{0}\left(1 \leq i_{0} \leq s\right)$ satisfy the condition in (10). For any index set $\alpha \subseteq N$, writing $\alpha=\alpha_{i_{1}} \cup \alpha_{i_{2}} \cup \cdots \cup \alpha_{i_{k}}$ and $\alpha^{c}=\alpha_{j_{1}} \cup \alpha_{j_{2}} \cup \cdots \cup \alpha_{j_{l}}$, with $k+l=s$, then:

(i) If $\alpha_{i_{0}} \subseteq \alpha$, then $A / \circ \alpha \in \mathrm{II}-\mathrm{BSD}_{l}$,

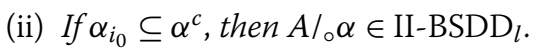

Proof Without loss of generality, we can assume $A /{ }_{\circ} \alpha=\left(\tilde{A}\left(\alpha_{t}, \alpha_{r}\right)\right)$, and we denote

$$
\begin{aligned}
& \Phi_{\omega}=\left(A\left(\alpha_{j_{\omega}}, \alpha_{i_{1}}\right), \ldots, A\left(\alpha_{j_{\omega}}, \alpha_{i_{k}}\right)\right)[A(\alpha)]^{-1}\left(\begin{array}{c}
A\left(\alpha_{i_{1}}, \alpha_{j_{\omega}}\right) \\
\vdots \\
A\left(\alpha_{i_{k}}, \alpha_{j_{\omega}}\right)
\end{array}\right), \\
& K_{\omega}=\left(\left\|\left[A\left(\alpha_{j_{\omega}}, \alpha_{i_{1}}\right)\right]^{-1} A\left(\alpha_{j_{\omega}}, \alpha_{i_{1}}\right)\right\|, \ldots,\left\|\left[A\left(\alpha_{j_{\omega}}, \alpha_{i_{1}}\right)\right]^{-1} A\left(\alpha_{j_{\omega}}, \alpha_{i_{k}}\right)\right\|\right), \\
& H_{\omega}=\left(\left\|\left[A\left(\alpha_{i_{1}}, \alpha_{i_{1}}\right)\right]^{-1} A\left(\alpha_{i_{1}}, \alpha_{j_{\omega}}\right)\right\|, \ldots,\left\|\left[A\left(\alpha_{i_{1}}, \alpha_{i_{1}}\right)\right]^{-1} A\left(\alpha_{i_{k}}, \alpha_{j_{\omega}}\right)\right\|\right)^{T}, \\
& D_{1}=\operatorname{diag}\left(A\left(\alpha_{i_{1}}, \alpha_{i_{1}}\right), \ldots, A\left(\alpha_{i_{k}}, \alpha_{i_{k}}\right)\right), \\
& \Psi_{\omega}=K_{\omega} \cdot \mu_{I I}\left\{[A(\alpha)]^{-1} D_{1}\right\} \cdot H_{\omega}, \quad \Upsilon_{\omega, v}=K_{\omega} \cdot\left\{\mu_{I I}[A(\alpha)] D_{1}^{-1}\right\}^{-1} \cdot H_{v}, \\
& \text { with } \omega, v=t, u .
\end{aligned}
$$

(i) If $\alpha_{i_{0}} \subseteq \alpha$, according to the proof of Theorem 3.1(i), for $\forall t \in \alpha^{c}$, we obtain

$$
\begin{aligned}
1- & \sum_{\substack{r=1 \\
r \neq t}}^{l}\left\|\left[\tilde{A}\left(\alpha_{t}, \alpha_{t}\right)\right]^{-1} \tilde{A}\left(\alpha_{t}, \alpha_{r}\right)\right\| \\
= & 1-\sum_{\substack{r=1 \\
r \neq t}}^{l}\left\|\left[A\left(\alpha_{j_{t}}, \alpha_{j_{t}}\right)-\Phi_{t}\right]^{-1} A\left(\alpha_{j_{t}}, \alpha_{j_{r}}\right)\right\|
\end{aligned}
$$




$$
\begin{aligned}
= & 1-\sum_{\substack{r=1 \\
r \neq t}}^{l}\left\|\left\{I_{J_{t}}-\left[A\left(\alpha_{j_{t}}, \alpha_{j_{t}}\right)\right]^{-1} \Phi_{t}\right\}^{-1}\left[A\left(\alpha_{j_{t}}, \alpha_{j_{t}}\right)\right]^{-1} A\left(\alpha_{j_{t}}, \alpha_{j_{r}}\right)\right\| \\
\geq & 1-\sum_{\substack{r=1 \\
r \neq t}}^{l}\left\|\left\{I_{J_{t}}-\left[A\left(\alpha_{j_{t}}, \alpha_{j_{t}}\right)\right]^{-1} \Phi_{t}\right\}^{-1}\right\|\left\|\left[A\left(\alpha_{j_{t}}, \alpha_{j_{t}}\right)\right]^{-1} A\left(\alpha_{j_{t}}, \alpha_{j_{r}}\right)\right\| \\
\geq & 1-\sum_{\substack{r=1 \\
r \neq t}}^{l}\left\{1-\left\|\left[A\left(\alpha_{j_{t}}, \alpha_{j_{t}}\right)\right]^{-1} \Phi_{t}\right\|\right\}^{-1}\left\|\left[A\left(\alpha_{j_{t}}, \alpha_{j_{t}}\right)\right]^{-1} A\left(\alpha_{j_{t}}, \alpha_{j_{r}}\right)\right\| \\
= & \left\{1-\left\|\left[A\left(\alpha_{j_{t}}, \alpha_{j_{t}}\right)\right]^{-1} \Phi_{t}\right\|\right\}^{-1} \\
& \times\left\{1-\left\|\left[A\left(\alpha_{j_{t}}, \alpha_{j_{t}}\right)\right]^{-1} \Phi_{t}\right\|-\sum_{\substack{r=1 \\
r \neq t}}^{l}\left\|\left[A\left(\alpha_{j_{t}}, \alpha_{j_{t}}\right)\right]^{-1} A\left(\alpha_{j_{t}}, \alpha_{j_{r}}\right)\right\|\right\} \\
\geq & \left\{1-\left\|\left[A\left(\alpha_{j_{t}}, \alpha_{j_{t}}\right)\right]^{-1} \Phi_{t}\right\|\right\}^{-1}\left\{1-\Upsilon_{t t}-\sum_{\substack{r=1 \\
r \neq t}}^{l}\left\|\left[A\left(\alpha_{j_{t}}, \alpha_{j_{t}}\right)\right]^{-1} A\left(\alpha_{j_{t}}, \alpha_{j_{r}}\right)\right\|\right\} \\
\triangleq & \frac{\operatorname{det} B_{3}}{\left(1-\left\|\left[A\left(\alpha_{j_{t}}, \alpha_{j_{t}}\right)\right]^{-1} \Phi_{t}\right\|\right) \operatorname{det}\left[\mu_{I I}(A)(\alpha)\right]},
\end{aligned}
$$

where

$$
B_{3}=\left(\begin{array}{cc}
1-\sum_{\substack{r=1 \\
r \neq t}}^{l}\left\|\left[A\left(\alpha_{j_{t}}, \alpha_{j_{t}}\right)\right]^{-1} A\left(\alpha_{j_{t}}, \alpha_{j_{r}}\right)\right\| & -K_{t} \\
-H_{t} & \mu_{I I}(A)(\alpha)
\end{array}\right) .
$$

Since $A$ is an $\mathrm{II}-\mathrm{BSDD}_{s}, \alpha_{i_{0}} \subseteq \alpha$, and $\alpha_{j_{t}} \subseteq \alpha^{c}$,

$$
1-\sum_{\substack{r=1 \\ r \neq t}}^{l}\left\|\left[A\left(\alpha_{j_{t}}, \alpha_{j_{t}}\right)\right]^{-1} A\left(\alpha_{j_{t}}, \alpha_{j_{r}}\right)\right\|>\sum_{r=1}^{k}\left\|\left[A\left(\alpha_{j_{t}}, \alpha_{j_{t}}\right)\right]^{-1} A\left(\alpha_{j_{t}}, \alpha_{i_{r}}\right)\right\| .
$$

For $\forall \alpha_{i_{x}} \subseteq \alpha, x=1,2, \ldots, k$, if $i_{x} \neq i_{0}$, then

$$
\begin{gathered}
\left\|\left[A\left(\alpha_{i_{x}}, \alpha_{i_{x}}\right)\right]^{-1} A\left(\alpha_{i_{x}}, \alpha_{j_{t}}\right)\right\|+\sum_{\substack{r=1 \\
r \neq x}}^{k}\left\|\left[A\left(\alpha_{i_{x}}, \alpha_{i_{x}}\right)\right]^{-1} A\left(\alpha_{i_{x}}, \alpha_{i_{r}}\right)\right\| \\
\quad \leq \sum_{\substack{r=1 \\
r \neq i_{x}}}^{s}\left\|\left[A\left(\alpha_{i_{x}}, \alpha_{i_{x}}\right)\right]^{-1} A\left(\alpha_{i_{x}}, \alpha_{r}\right)\right\|<1, \\
\end{gathered}
$$

if $i_{x}=i_{0}$, by Definition 2.4 and the inequality (10), we have

$$
\begin{aligned}
1- & \sum_{\substack{r=1 \\
r \neq t}}^{l}\left\|\left[A\left(\alpha_{j_{t}}, \alpha_{j_{t}}\right)\right]^{-1} A\left(\alpha_{j_{t}}, \alpha_{j_{r}}\right)\right\| \\
& \geq 1-\sum_{\substack{r=1 \\
r \neq t}}^{l}\left\|\left[A\left(\alpha_{j_{t}}, \alpha_{j_{t}}\right)\right]^{-1} A\left(\alpha_{j_{t}}, \alpha_{j_{r}}\right)\right\| \sum_{\substack{r=1 \\
r \neq i_{0}}}^{s}\left\|\left[A\left(\alpha_{i_{0}}, \alpha_{i_{0}}\right)\right]^{-1} A\left(\alpha_{i_{0}}, \alpha_{r}\right)\right\|
\end{aligned}
$$




$$
\begin{aligned}
& >\sum_{r=1}^{k}\left\|\left[A\left(\alpha_{j_{t}}, \alpha_{j_{t}}\right)\right]^{-1} A\left(\alpha_{j_{t}}, \alpha_{j_{r}}\right)\right\| \sum_{\substack{r=1 \\
r \neq i_{0}}}^{s}\left\|\left[A\left(\alpha_{i_{0}}, \alpha_{i_{0}}\right)\right]^{-1} A\left(\alpha_{i_{0}}, \alpha_{r}\right)\right\| \\
& \geq \sum_{r=1}^{k}\left\|\left[A\left(\alpha_{j_{t}}, \alpha_{j_{t}}\right)\right]^{-1} A\left(\alpha_{j_{t}}, \alpha_{j_{r}}\right)\right\| \\
& \quad \times\left\{\left\|\left[A\left(\alpha_{i_{0}}, \alpha_{i_{0}}\right)\right]^{-1} A\left(\alpha_{i_{0}}, \alpha_{j_{t}}\right)\right\|+\sum_{\substack{r=1 \\
i_{r} \neq i_{0}}}^{k}\left\|\left[A\left(\alpha_{i_{0}}, \alpha_{i_{0}}\right)\right]^{-1} A\left(\alpha_{i_{0}}, \alpha_{i_{r}}\right)\right\|\right\} .
\end{aligned}
$$

Thus, $B_{3} \in \operatorname{SDD}_{k+1}$. Further, by Lemma 2.3, we obtain $B_{3}=\mu\left(B_{1}\right) \in M_{k+1}$ and $\mu_{I I}[A(\alpha)] \in$ $M_{k}$. Therefore, $\operatorname{det}\left(B_{3}\right)>0$ and $\operatorname{det}\left[\mu_{I I}(A(\alpha))\right]>0$, i.e.,

$A / \circ \alpha \in{\mathrm{II}-\mathrm{BSD}_{l}}$

(ii) If $\alpha_{i_{0}} \subseteq \alpha^{c}$, for $\forall t, u \in \alpha^{c}$, with $t, u=1,2, \ldots, l, t \neq u$, we obtain

$$
\begin{aligned}
& 1-\sum_{\substack{r=1 \\
r \neq t}}^{l}\left\|\left[\tilde{A}\left(\alpha_{t}, \alpha_{t}\right)\right]^{-1} \tilde{A}\left(\alpha_{t}, \alpha_{r}\right)\right\| \sum_{\substack{r=1 \\
r \neq u}}^{l}\left\|\left[\tilde{A}\left(\alpha_{u}, \alpha_{u}\right)\right]^{-1} \tilde{A}\left(\alpha_{u}, \alpha_{r}\right)\right\| \\
& =1-\sum_{\substack{r=1 \\
r \neq t}}^{l}\left\|\left[A\left(\alpha_{j_{t}}, \alpha_{j_{t}}\right)-\Phi_{t}\right]^{-1} A\left(\alpha_{j_{t}}, \alpha_{j_{r}}\right)\right\| \sum_{\substack{r=1 \\
r \neq u}}^{l}\left\|\left[A\left(\alpha_{j_{u}}, \alpha_{j_{u}}\right)-\Phi_{u}\right]^{-1} A\left(\alpha_{j_{u}}, \alpha_{j_{r}}\right)\right\| \\
& =1-\sum_{\substack{r=1 \\
r \neq t}}^{l}\left\|\left\{I_{J_{t}}-\left[A\left(\alpha_{j_{t}}, \alpha_{j_{t}}\right)\right]^{-1} \Phi_{t}\right\}^{-1}\left[A\left(\alpha_{j_{t}}, \alpha_{j_{t}}\right)\right]^{-1} A\left(\alpha_{j_{t}}, \alpha_{j_{r}}\right)\right\| \\
& \times \sum_{\substack{r=1 \\
r \neq u}}^{l}\left\|\left\{I_{J_{u}}-\left[A\left(\alpha_{j_{u}}, \alpha_{j_{u}}\right)\right]^{-1} \Phi_{u}\right\}^{-1}\left[A\left(\alpha_{j_{u}}, \alpha_{j_{u}}\right)\right]^{-1} A\left(\alpha_{j_{u}}, \alpha_{j_{r}}\right)\right\| \\
& \geq 1-\sum_{\substack{r=1 \\
r \neq t}}^{l}\left\|\left\{I_{J_{t}}-\left[A\left(\alpha_{j_{t}}, \alpha_{j_{t}}\right)\right]^{-1} \Phi_{t}\right\}^{-1}\right\|\left\|\left[A\left(\alpha_{j_{t}}, \alpha_{j_{t}}\right)\right]^{-1} A\left(\alpha_{j_{t}}, \alpha_{j_{r}}\right)\right\| \\
& \times \sum_{\substack{r=1 \\
r \neq t}}^{l}\left\|\left\{I_{J_{u}}-\left[A\left(\alpha_{j_{u}}, \alpha_{j_{u}}\right)\right]^{-1} \Phi_{u}\right\}^{-1}\right\|\left\|\left[A\left(\alpha_{j_{u}}, \alpha_{j_{u}}\right)\right]^{-1} A\left(\alpha_{j_{u}}, \alpha_{j_{r}}\right)\right\| \\
& \geq 1-\sum_{\substack{r=1 \\
r \neq t}}^{l}\left\{1-\left\|\left[A\left(\alpha_{j_{t}}, \alpha_{j_{t}}\right)\right]^{-1} \Phi_{t}\right\|\right\}^{-1}\left\|\left[A\left(\alpha_{j_{t}}, \alpha_{j_{t}}\right)\right]^{-1} A\left(\alpha_{j_{t}}, \alpha_{j_{r}}\right)\right\| \\
& \times \sum_{\substack{r=1 \\
r \neq u}}^{l}\left\{1-\left\|\left[A\left(\alpha_{j_{u}}, \alpha_{j_{u}}\right)\right]^{-1} \Phi_{u}\right\|\right\}^{-1}\left\|\left[A\left(\alpha_{j_{u}}, \alpha_{j_{u}}\right)\right]^{-1} A\left(\alpha_{j_{u}}, \alpha_{j_{r}}\right)\right\| \\
& =\left\{1-\left\|\left[A\left(\alpha_{j_{t}}, \alpha_{j_{t}}\right)\right]^{-1} \Phi_{t}\right\|\right\}^{-1}\left\{1-\left\|\left[A\left(\alpha_{j_{u}}, \alpha_{j_{u}}\right)\right]^{-1} \Phi_{u}\right\|\right\}^{-1} \\
& \times\left\{\left[1-\left\|\left[A\left(\alpha_{j_{t}}, \alpha_{j_{t}}\right)\right]^{-1} \Phi_{t}\right\|\right]\left[1-\left\|\left[A\left(\alpha_{j_{u}}, \alpha_{j_{u}}\right)\right]^{-1} \Phi_{u}\right\|\right]\right.
\end{aligned}
$$




$$
\begin{aligned}
& \left.-\sum_{\substack{r=1 \\
r \neq t}}^{l}\left\|\left[A\left(\alpha_{j_{t}}, \alpha_{j_{t}}\right)\right]^{-1} A\left(\alpha_{j_{t}}, \alpha_{j_{r}}\right)\right\| \sum_{\substack{r=1 \\
r \neq u}}^{l}\left\|\left[A\left(\alpha_{j_{u}}, \alpha_{j_{u}}\right)\right]^{-1} A\left(\alpha_{j_{u}}, \alpha_{j_{r}}\right)\right\|\right\} \\
\geq & \left\{1-\left\|\left[A\left(\alpha_{j_{t}}, \alpha_{j_{t}}\right)\right]^{-1} \Phi_{t}\right\|\right\}^{-1}\left\{1-\left\|\left[A\left(\alpha_{j_{u}}, \alpha_{j_{u}}\right)\right]^{-1} \Phi_{u}\right\|\right\}^{-1}\left\{\left(1-\Upsilon_{t t}\right)\left(1-\Upsilon_{u u}\right)\right. \\
& \left.-\left[\sum_{\substack{r=1 \\
r \neq t}}^{l}\left\|\left[A\left(\alpha_{j_{t}}, \alpha_{j_{t}}\right)\right]^{-1} A\left(\alpha_{j_{t}}, \alpha_{j_{r}}\right)\right\|+\Upsilon_{t u}\right]\left[\sum_{\substack{r=1 \\
r \neq u}}^{l}\left\|\left[A\left(\alpha_{j_{u}}, \alpha_{j_{u}}\right)\right]^{-1} A\left(\alpha_{j_{u}}, \alpha_{j_{r}}\right)\right\|+\Upsilon_{u t}\right]\right\} \\
= & \left\{1-\left\|\left[A\left(\alpha_{j_{t}}, \alpha_{j_{t}}\right)\right]^{-1} \Phi_{t}\right\|\right\}^{-1}\left\{1-\left\|\left[A\left(\alpha_{j_{u}}, \alpha_{j_{u}}\right)\right]^{-1} \Phi_{u}\right\|\right\}^{-1} \\
\triangleq & \frac{\operatorname{det} B_{4}}{\operatorname{det}\left[\mu_{I I}(A)(\alpha)\right]},
\end{aligned}
$$

where

$$
B_{4}=\left(\begin{array}{ccc}
1 & -\xi_{t} & -K_{t} \\
-\xi_{u} & 1 & -K_{u} \\
-H_{t} & -H_{u} & \mu_{I I}(A)(\alpha)
\end{array}\right), \quad \xi_{\omega}=\sum_{\substack{r=1 \\
r \neq \omega}}^{l}\left\|\left[A\left(\alpha_{j_{\omega}}, \alpha_{j_{\omega}}\right)\right]^{-1} A\left(\alpha_{j_{\omega}}, \alpha_{j_{r}}\right)\right\|,
$$

with $\omega=t$, $u$.

Since $A$ is an II-BSDD $s, \alpha_{i_{0}} \subseteq \alpha^{c}$, and $\alpha_{j_{\omega}} \subseteq \alpha^{c}, \omega=t, u$,

$$
\sum_{\substack{r=1 \\ r \neq j t}}^{s}\left\|\left[A\left(\alpha_{j_{t}}, \alpha_{j_{t}}\right)\right]^{-1} A\left(\alpha_{j_{t}}, \alpha_{r}\right)\right\| \sum_{\substack{r=1 \\ r \neq j_{u}}}^{s}\left\|\left[A\left(\alpha_{j_{u}}, \alpha_{j_{u}}\right)\right]^{-1} A\left(\alpha_{j_{u}}, \alpha_{r}\right)\right\|<1 .
$$

For $\forall \alpha_{i_{x}} \subseteq \alpha, x=1,2, \ldots, k$, and $\alpha_{j_{w}} \subseteq \alpha^{c}, w=t, u$.

$$
\begin{aligned}
& \left\{\sum_{\substack{r=1 \\
r \neq x}}^{s}\left\|\left[A\left(\alpha_{i_{x}}, \alpha_{i_{x}}\right)\right]^{-1} A\left(\alpha_{i_{x}}, \alpha_{i_{r}}\right)\right\|+\left\|\left[A\left(\alpha_{i_{x}}, \alpha_{i_{x}}\right)\right]^{-1} A\left(\alpha_{i_{x}}, \alpha_{j_{t}}\right)\right\|\right. \\
& \left.\quad+\left\|\left[A\left(\alpha_{i_{x}}, \alpha_{i_{x}}\right)\right]^{-1} A\left(\alpha_{i_{x}}, \alpha_{j_{u}}\right)\right\|\right\} \sum_{\substack{r=1 \\
r \neq j_{\omega}}}^{s}\left\|\left[A\left(\alpha_{j_{\omega}}, \alpha_{j_{\omega}}\right)\right]^{-1} A\left(\alpha_{j_{\omega}}, \alpha_{r}\right)\right\|<1 .
\end{aligned}
$$

For $\forall \alpha_{i_{x}} \subseteq \alpha$, with $x=1,2, \ldots, k$,

$$
\begin{aligned}
& \left\{\sum_{\substack{r=1 \\
r \neq x}}^{k}\left\|\left[A\left(\alpha_{i_{x}}, \alpha_{i_{x}}\right)\right]^{-1} A\left(\alpha_{i_{x}}, \alpha_{i_{r}}\right)\right\|+\left\|\left[A\left(\alpha_{i_{x}}, \alpha_{i_{x}}\right)\right]^{-1} A\left(\alpha_{i_{x}}, \alpha_{j_{u}}\right)\right\|\right. \\
& \left.\quad+\left\|\left[A\left(\alpha_{i_{x}}, \alpha_{i_{x}}\right)\right]^{-1} A\left(\alpha_{i_{x}}, \alpha_{j_{t}}\right)\right\|\right\}<1 .
\end{aligned}
$$

Thus, $B_{4} \in \mathrm{SDD}_{k+2}$. Further, by Lemma 2.3, we have $B_{4}=\mu\left(B_{4}\right) \in M_{k+2}, \mu_{I I}[A(\alpha)] \in M_{k}$. Therefore, $\operatorname{det}\left(B_{4}\right)>0$ and $\operatorname{det}\left[\mu_{I I}(A)(\alpha)\right]>0$, i.e.,

$$
A /{ }_{\circ} \alpha \in \mathrm{II}-\mathrm{BSDD}_{l}
$$

Combining the proof of (i) and (ii), we complete this proof. 


\section{Numerical examples}

In this section, we use two numerical examples to verify the accuracy of the theoretical analysis, from two aspects of the iteration number (denoted by IT) and the solution time in seconds (denoted by CPU), and then we further illustrate the feasibility and effectiveness of PGMSS $(l)$ [19] iteration method, and verify the superiority of PGMSS iteration method is more efficient than that of the ordinary $\operatorname{GMRES}(l)$ iteration method. Here, we use the integer $l$ in GMRES $(l)$ to denote the number of restarting steps.

In our numerical experiments, we choose the zero vector as the initial guess and take the right-hand-side vector $b$ so that the exact solutions $x$ and $y$ are the unity vectors with all entries equal to one. In addition, all runs are initiated with the initial vector $x^{(0)}=0$. We use

$$
\mathrm{RES}=\frac{\left\|b-A x^{(k)}\right\|_{2}}{\left\|b-A x^{(0)}\right\|_{2}}<10^{-8}
$$

or the prescribed iteration number $k_{\max }=n$ as a stopping criterion, where $x^{(k)}$ is the solution at the $k$ th iterate.

For convenience, without loss of generality, we suppose $\|\cdot\|=\|\cdot\|_{2}, \alpha=\bigcup_{r=1}^{3} \alpha_{i_{r}}$ and $\alpha^{c}=\bigcup_{t=1}^{2} \alpha_{j t}$, and we denote the block diagonal-Schur complement $A /{ }_{\circ} A(\alpha)$ of $A(\alpha)$ in $A$ by

$$
A / \circ A(\alpha)=\left(\begin{array}{ccc}
\widetilde{A}_{11} & \widetilde{A}_{12} & \widetilde{A}_{13} \\
\widetilde{A}_{21} & \widetilde{A}_{22} & \widetilde{A}_{23} \\
\widetilde{A}_{31} & \widetilde{A}_{32} & \widetilde{A}_{33}
\end{array}\right) .
$$

Let us consider the following linear system:

$$
A x=\left(\begin{array}{ll}
A(\alpha, \alpha) & A\left(\alpha, \alpha^{c}\right) \\
A\left(\alpha^{c}, \alpha\right) & A\left(\alpha^{c}, \alpha^{c}\right)
\end{array}\right)\left(\begin{array}{l}
x_{1} \\
x_{2}
\end{array}\right)=\left(\begin{array}{l}
b_{1} \\
b_{2}
\end{array}\right) .
$$

By solving the above linear system, we compare GMRES iteration method with the block triangular approximate Schur complement preconditioner (11) established in this paper with the ordinary GMRES iteration method. We have

$$
P_{1}=\left(\begin{array}{ll}
A(\alpha, \alpha) & \\
A\left(\alpha^{c}, \alpha\right) & A /{ }_{\circ} A(\alpha)
\end{array}\right) .
$$

In the following, we verify Theorem 3.1 and Theorem 3.2 by Example 4.1 and Example 4.2 , respectively.

Example 4.1 Consider a linear equation system $A x=b$ whose coefficient matrix $A$ is denoted by

$$
A=\left(\begin{array}{lllll}
A_{11} & A_{12} & A_{13} & A_{14} & A_{15} \\
A_{21} & A_{22} & A_{23} & A_{24} & A_{25} \\
A_{31} & A_{32} & A_{33} & A_{34} & A_{35} \\
A_{41} & A_{42} & A_{43} & A_{44} & A_{45} \\
A_{51} & A_{52} & A_{53} & A_{54} & A_{55}
\end{array}\right),
$$


where the submatrices of the coefficient matrix $A$ take on structures of the forms

$$
\begin{aligned}
& A_{11}=\left(\begin{array}{ccccc}
150 & -50 & & & \\
-50 & 150 & -50 & & \\
& \ddots & \ddots & \ddots & \\
& & & -50 & 150
\end{array}\right)_{20 \times 20} \\
& A_{22}=\left(\begin{array}{ccccc}
1,500 & -50 & & & \\
-50 & 1,500 & -50 & & \\
& \ddots & \ddots & \ddots & \\
& & & -50 & 1,500
\end{array}\right)_{20 \times 20}, \\
& A_{33}=\left(\begin{array}{ccccc}
1,500 & -50 & & & \\
-50 & 1,500 & -50 & & \\
& \ddots & \ddots & \ddots & \\
& & & -50 & 1,500
\end{array}\right)_{30 \times 30}, \\
& A_{44}=\left(\begin{array}{ccccc}
1,500 & -50 & & & \\
-50 & 1,500 & -50 & & \\
& \ddots & \ddots & \ddots & \\
& & & -50 & 1,500
\end{array}\right)_{15 \times 15} \text {, }
\end{aligned}
$$

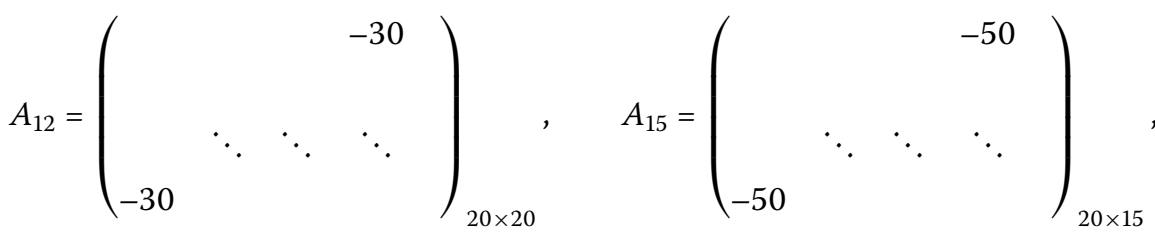

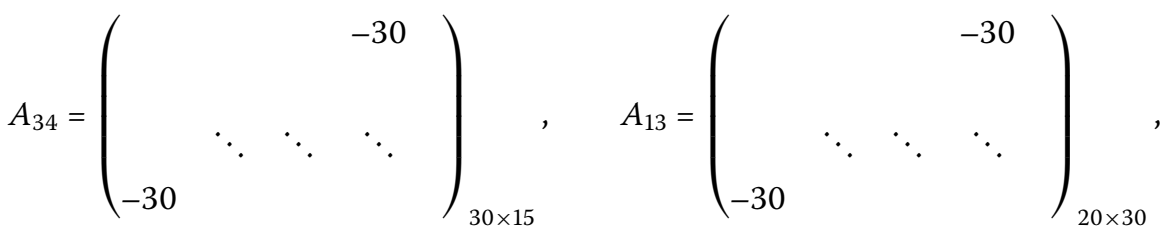

and

$$
A_{45}=\left(\begin{array}{cccc}
-20 & & & -20 \\
& \ddots & \ddots & \ddots \\
-20 & & & -20
\end{array}\right)_{15 \times 15},
$$

with $A_{44}=A_{55}, A_{21}^{T}=A_{12}, A_{14}=A_{15}=A_{24}=A_{25}=A_{41}^{T}=A_{51}^{T}=A_{42}^{T}=A_{52}^{T}, A_{54}^{T}=A_{45}, A_{43}^{T}=$ $A_{34}=A_{53}^{T}=A_{35}$, and $A_{31}^{T}=A_{13}=A_{32}^{T}=A_{23}$.

We suppose $t_{i}=\left\|\left[A_{i i}\right]^{-1}\right\|^{-1}$ and $s_{i}=\sum_{\substack{m=1 \\ m \neq i}}^{5}\left\|A_{i m}\right\|$, with $i=1,2, \ldots, 5$. After programming and computation by the use of Matlab software, from Table 1, it is straightforward to show that the matrix $A$ is an $\mathrm{I}-\mathrm{BSDD}_{s}$ but is not an $\mathrm{I}-\mathrm{BSD}_{s}$. 
Table 1 The experimental verification of I-BSDD

\begin{tabular}{llllll}
\hline $\mathbf{i}$ & $\mathbf{1}$ & $\mathbf{2}$ & $\mathbf{3}$ & $\mathbf{4}$ & $\mathbf{5}$ \\
\hline$t_{i}-s_{i}$ & $\mathbf{- 1 0 8 . 8 8}$ & $1.24 \times 10^{3}$ & $3.97 \times 10^{3}$ & $1.23 \times 10^{3}$ & $1.23 \times 10^{3}$ \\
\hline $\mathbf{( i , j )}$ & $\mathbf{( 1 , 2 )}$ & $\mathbf{( 1 , 3 )}$ & $\mathbf{( 1 , 4 )}$ & $\mathbf{( 1 , 5 )}$ & $\mathbf{( 2 , 3 )}$ \\
\hline$t_{i} t_{j}-s_{i} s_{j}$ & $4.6 \times 10^{4}$ & $5.24 \times 10^{4}$ & $4.45 \times 10^{4}$ & $4.45 \times 10^{4}$ & $1.94 \times 10^{6}$ \\
\hline $\mathbf{( i , j )}$ & $\mathbf{( 2 , 4 )}$ & $\mathbf{( 2 , 5 )}$ & $\mathbf{( 3 , 4 )}$ & $\mathbf{( 3 , 5 )}$ & $\mathbf{( 4 , 5 )}$ \\
\hline$t_{i} t_{j}-s_{i} s_{j}$ & $1.94 \times 10^{6}$ & $1.94 \times 10^{6}$ & $1.94 \times 10^{6}$ & $1.94 \times 10^{6}$ & $1.94 \times 10^{6}$ \\
\hline
\end{tabular}

Table 2 The experimental verification of I-BSD $s$

\begin{tabular}{llll}
\hline $\boldsymbol{i}$ & $\mathbf{1}$ & $\mathbf{2}$ & $\mathbf{3}$ \\
\hline$\widetilde{t}_{i}-\widetilde{s}_{i}$ & 1,341 & 1,332 & 1,332 \\
$\widetilde{t}_{i} \tilde{t}_{j}-\widetilde{s}_{i} \widetilde{s}_{j}$ & $1.96 \times 10^{6}$ & $1.96 \times 10^{6}$ & $1.96 \times 10^{6}$ \\
\hline
\end{tabular}

Table 3 The experimental verification of $\mathrm{I-BSD}_{s}$

\begin{tabular}{llll}
\hline $\boldsymbol{i}$ & $\mathbf{1}$ & $\mathbf{2}$ & $\mathbf{3}$ \\
\hline$\widehat{t}_{i}-\widehat{s}_{i}$ & $\mathbf{- 8 . 8 9 8 5}$ & 1,341 & 1,341 \\
$\widehat{t}_{i} t_{j}-\widehat{s}_{i} \hat{s}_{j}$ & $6.8 \times 10^{4}$ & $6.8 \times 10^{4}$ & $1.96 \times 10^{6}$ \\
\hline
\end{tabular}

Table 4 Number of iterations and solution time in seconds of PGMRES $(l)$ iteration method with preconditioner $\mathcal{P}_{1}$ and the ordinary $\operatorname{GMRES}(I)$ iteration method, where $\alpha=\{1,2\}$

\begin{tabular}{lllllllll}
\hline $\boldsymbol{I}$ & & $\mathbf{3 0}$ & $\mathbf{4 0}$ & $\mathbf{5 0}$ & $\mathbf{6 0}$ & $\mathbf{7 0}$ & $\mathbf{8 0}$ & $\mathbf{9 0}$ \\
\hline PGMRES(I) & CPU & 0.0106 & 0.0015 & 0.0024 & 0.0018 & 0.0014 & 0.0024 & 0.0015 \\
& IT & 4 & 4 & 4 & 4 & 4 & 4 & 4 \\
\multirow{2}{*}{ GMRES(I) } & CPU & 0.0046 & 0.0054 & 0.0042 & 0.0018 & 0.0042 & 0.0156 & 0.0048 \\
& IT & 23 & 23 & 23 & 23 & 23 & 23 & 23 \\
\hline
\end{tabular}

To verify the heritable properties of the block diagonal-Schur complements from the original matrix I-BSDD , we need to consider two conditions $i_{0} \in \alpha$ and $i_{0} \in \alpha^{c}$, where $i_{0}$ is defined in (9). From Table 1 , it is easy to see $i_{0}=1$. Firstly, we consider the condition $i_{0} \in \alpha=$ $\{1,2\}$ and $\alpha^{c}=\{3,4,5\}$, and assume $\widetilde{t}_{i}=\left\|\left[\widetilde{A}_{i i}\right]^{-1}\right\|^{-1}$ and $\widetilde{s}_{i}=\sum_{\substack{m=1 \\ m \neq i}}^{3}\left\|\widetilde{A}_{i m}\right\|$, with $i=1,2,3$, as follows from Table 2 , we can easily see that the block diagonal-Schur complement $A /{ }_{\circ} A(\alpha)$ of $A(\alpha)$ in $A$ is an $\mathrm{I}-\mathrm{BSD}_{s}$; thereby, we verify the conclusion (i) of Theorem 3.1. Secondly, we consider the condition $i_{0} \in \alpha^{c}=\{1,2,3\}$ and $\alpha=\{4,5\}$, and assume $\widehat{t}_{i}=\left\|\left[\widetilde{A}_{i i}\right]^{-1}\right\|^{-1}$ and $\widehat{s}_{i}=\sum_{\substack{m=1 \\ m \neq i}}^{3}\left\|\widetilde{A}_{i m}\right\|$, with $i=1,2,3$, as follows from Table 3 , we can easily see that the block diagonal-Schur complement $A /{ }_{\circ} A(\alpha)$ of $A(\alpha)$ in $A$ is an $\mathrm{I}-\mathrm{BSDD}_{s}$ but is not an $\mathrm{I}_{-} \mathrm{BSD}_{s}$, accordingly, we validate the conclusion (ii) of Theorem 3.1.

Table 4, for Example 4.1, lists the numerical results corresponding to the tolerance $\epsilon=$ $10^{-8}$, it means that the block diagonal Schur-based GMRES iteration method with the preconditioner $P_{1}$ is more efficient than the ordinary GMRES $(l)$ iteration method. 
Example 4.2 Consider a linear equation system $A x=b$ whose coefficient matrix $A$ is denoted by

$$
A=\left(\begin{array}{lllll}
A_{11} & A_{12} & A_{13} & A_{14} & A_{15} \\
A_{21} & A_{22} & A_{23} & A_{24} & A_{25} \\
A_{31} & A_{32} & A_{33} & A_{34} & A_{35} \\
A_{41} & A_{42} & A_{43} & A_{44} & A_{45} \\
A_{51} & A_{52} & A_{53} & A_{54} & A_{55}
\end{array}\right),
$$

where the submatrices of the coefficient matrix $A$ take on structures of the forms

$$
\begin{aligned}
& A_{11}=\left(\begin{array}{ccccc}
100 & -50 & & & \\
-50 & 100 & -50 & & \\
& \ddots & \ddots & \ddots & \\
& & & -50 & 100
\end{array}\right)_{20 \times 20} \\
& A_{22}=\left(\begin{array}{ccccc}
1,800 & -50 & & & \\
-50 & 1,800 & -50 & & \\
& \ddots & \ddots & \ddots & \\
& & & -50 & 1,800
\end{array}\right)_{20 \times 20}, \\
& A_{33}=\left(\begin{array}{ccccc}
1,410 & -40 & & & \\
-40 & 1,420 & -40 & & \\
& \ddots & \ddots & \ddots & \\
& & & -40 & 1,700
\end{array}\right)_{30 \times 30}, \\
& A_{44}=\left(\begin{array}{ccccc}
1,800 & -50 & & & \\
-50 & 1,800 & -50 & & \\
& \ddots & \ddots & \ddots & \\
& & & -50 & 1,800
\end{array}\right)_{15 \times 15},
\end{aligned}
$$

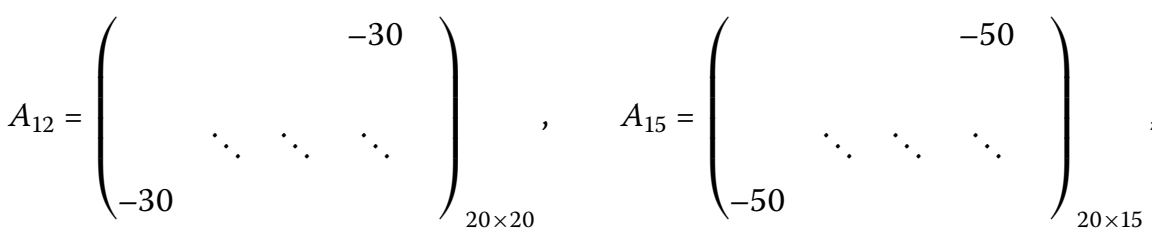

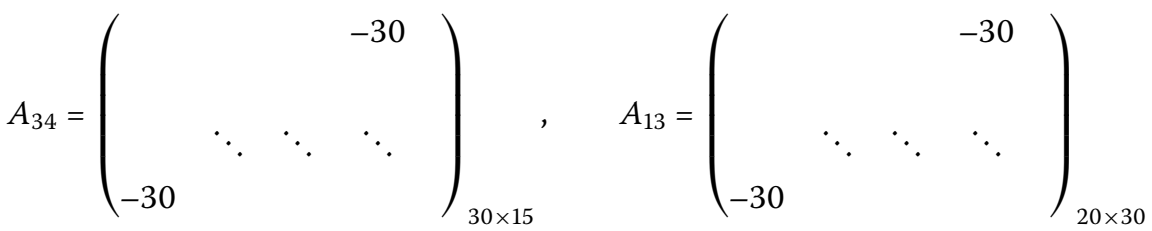

and

$$
A_{45}=\left(\begin{array}{cccc}
-20 & & & -20 \\
& \ddots & \ddots & \ddots \\
-20 & & & -20
\end{array}\right)_{15 \times 15},
$$


Table 5 The experimental verification of ${\mathrm{I}-\mathrm{BSDD}_{s}}$

\begin{tabular}{llllll}
\hline $\boldsymbol{i}$ & $\mathbf{1}$ & $\mathbf{2}$ & $\mathbf{3}$ & $\mathbf{4}$ & $\mathbf{5}$ \\
\hline$\zeta_{i}$ & $\mathbf{1 0 . 1 2}$ & 0.089 & 0.085 & 0.095 & 0.095 \\
\hline $\mathbf{( i , j )}$ & $\mathbf{( 1 , 2 )}$ & $\mathbf{( 1 , 3 )}$ & $\mathbf{( 1 , 4 )}$ & $\mathbf{( 1 , 5 )}$ & $\mathbf{( 2 , 3 )}$ \\
\hline$\zeta_{i} \zeta_{j}$ & 0.9005 & 0.8622 & 0.9568 & 0.9568 & 0.0076 \\
\hline $\mathbf{(}, \mathbf{j})$ & $\mathbf{( 2 , 4 )}$ & $\mathbf{( 2 , 5 )}$ & $\mathbf{( 3 , 4 )}$ & $\mathbf{( 3 , 5 )}$ & $\mathbf{( 4 , 5 )}$ \\
\hline$\zeta_{i} \zeta_{j}$ & 0.0084 & 0.0084 & 0.0081 & 0.0081 & 0.0089 \\
\hline
\end{tabular}

Table 6 The experimental verification of II-BSDD $s$

\begin{tabular}{llll}
\hline $\boldsymbol{i}$ & $\mathbf{1}$ & $\mathbf{2}$ & $\mathbf{3}$ \\
\hline$\widetilde{s}_{i}$ & 0.0432 & 0.0401 & 0.0401 \\
$\widetilde{s}_{i} \widetilde{s}_{j}$ & 0.0017 & 0.0017 & 0.0016 \\
\hline
\end{tabular}

Table 7 The experimental verification of II-BSDD $s$

\begin{tabular}{llll}
\hline $\boldsymbol{i}$ & $\mathbf{1}$ & $\mathbf{2}$ & $\mathbf{3}$ \\
\hline$\widehat{S}_{i}$ & $\mathbf{4 . 0 2 3 5}$ & 0.0334 & 0.0426 \\
$\widehat{S}_{i} \hat{S}_{j}$ & 0.1345 & 0.1715 & 0.0014 \\
\hline
\end{tabular}

Table 8 Number of iterations and solution time in seconds of PGMRES $(I)$ iteration method with preconditioner $\mathcal{P}_{1}$ and $\operatorname{GMRES}(I)$ iteration method

\begin{tabular}{lllllllll}
\hline $\boldsymbol{I}$ & & $\mathbf{3 0}$ & $\mathbf{4 0}$ & $\mathbf{5 0}$ & $\mathbf{6 0}$ & $\mathbf{7 0}$ & $\mathbf{8 0}$ & $\mathbf{9 0}$ \\
\hline PGMRES(I) & CPU & 0.0021 & 0.0026 & 0.0016 & 0.0016 & 0.0033 & 0.0021 & 0.0016 \\
& IT & 4 & 4 & 4 & 4 & 4 & 4 & 4 \\
\multirow{2}{*}{ GMRES(I) } & CPU & 0.0096 & 0.0159 & 0.0197 & 0.0141 & 0.0196 & 0.0186 & 0.0176 \\
& IT & 27 & 25 & 24 & 53 & 53 & 53 & 53 \\
\hline
\end{tabular}

with $A_{44}=A_{55}, A_{21}^{T}=A_{12}, A_{14}=A_{15}=A_{24}=A_{25}=A_{41}^{T}=A_{51}^{T}=A_{42}^{T}=A_{52}^{T}, A_{54}^{T}=A_{45}, A_{43}^{T}=$ $A_{34}=A_{53}^{T}=A_{35}$, and $A_{31}^{T}=A_{13}=A_{32}^{T}=A_{23}$.

After programming and computation by the use of Matlab software, denoting $\zeta_{i}=$ $\sum_{\substack{m=1 \\ m \neq i}}^{5}\left\|\left[A_{i i}\right]^{-1} A_{i m}\right\|$, with $i=1,2, \ldots, 5$, from Table 5 , it is straightforward to show that the matrix $A$ is an II-BSDD but is not an II-BSD .

To verify the heritable properties of the block diagonal-Schur complements from the original matrix II-BSDD , we need to consider two conditions $i_{0} \in \alpha$ and $i_{0} \in \alpha^{c}$, where $i_{0}$ is defined in (10). From the first line of Table 5 , it is easy to see that $i_{0}=1$. Firstly, we consider the condition $i_{0} \in \alpha=\{1,2\}$ and $\alpha^{c}=\{3,4,5\}$, and assume $\widetilde{s}_{i}=\sum_{\substack{m=1 \\ m \neq i}}^{3}\left\|\left[\widetilde{A}_{i i}\right]^{-1} \widetilde{A}_{i m}\right\|$, with $i=1,2,3$, as follows from Table 6, we can easily see that the block diagonal-Schur complement $A / \circ A(\alpha)$ of $A(\alpha)$ in $A$ is an $\mathrm{I}-\mathrm{BSD}_{s}$, therefore, we verify the result (i) of Theorem 3.2. Secondly, we consider the condition $i_{0} \in \alpha^{c}=\{1,2,3\}$ and $\alpha=\{4,5\}$, and assume $\widehat{s}_{i}=\sum_{\substack{m=1 \\ m \neq i}}^{3}\left\|\left[\widetilde{A}_{i i}\right]^{-1} \widetilde{A}_{i m}\right\|$, with $i=1,2,3$, as follows from Table 7 , we can easily see that the block diagonal-Schur complement $A /{ }_{\circ} A(\alpha)$ of $A(\alpha)$ in $A$ is an II-BSDD but is not an II-BSD ${ }_{s}$, accordingly, we validate the result (ii) of Theorem 3.2.

Table 8, for Example 4.2, lists the numerical results corresponding to the tolerance $\epsilon=$ $10^{-8}$, it means that the block diagonal Schur-based GMRES $(l)$ iteration method with the 
preconditioner $P_{1}$ is more efficient than the ordinary GMRES $(l)$ iteration method, where $\alpha=\{1,2\}$.

\section{Conclusions}

In this paper, the heritable properties of the block diagonal-Schur complements from the original matrix are presented. Numerical experiments further indicate the practical performance.

One of the advantages of the study of the heritable properties is that it is possible to estimate the sharp bounds of the eigenvalues of the original matrix and the corresponding block diagonal-Schur complements. Thereby we believe that the techniques presented here can be used to develop robust and efficient Schur complement preconditioning techniques for solving linear systems.

\section{Competing interests}

The author declares that he has no competing interests.

\section{Acknowledgements}

The author gratefully acknowledges the valuable comments and suggestions from the anonymous referees. This research was supported by the Project Foundation of Chongqing Municipal Education Committee (KJ120832).

Received: 29 September 2014 Accepted: 13 February 2015 Published online: 04 March 2015

\section{References}

1. Berman, A, Plemmons, RJ: Nonnegative Matrices in the Mathematical Sciences. Academic Press, New York (1979)

2. Calson, D, Markham, T: Schur complements on diagonally dominant matrices. Czechoslov. Math. J. 29, 246-251 (1979)

3. Liu, JZ, Huang, YQ: Some properties on Schur complements of $\mathrm{H}$-matrices and diagonally dominant matrices. Linear Algebra Appl. 389, 365-380 (2004)

4. Golub, GH, Van Loan, CF: Matrix Computations, 3rd edn. Johns Hopkins University Press, Baltimore (1996)

5. Kress, R: Numerical Analysis. Springer, New York (1998)

6. Song, YZ: The convergence of block AOR iterative methods. Appl. Math. 6, $39-45$ (1993)

7. Petra, CG, Anitescu, M: A preconditioning technique for Schur complement systems arising in stochastic optimization. Comput. Optim. Appl. 52, 315-344 (2012)

8. Malas, T, Gürel, L: Schur complement preconditioners for surface integral-equation formulations of dielectric problems solved with the multilevel fast multipole algorithm. SIAM J. Sci. Comput. 33, 2440-2467 (2011)

9. Yamazaki, I, Ng, EG: Preconditioning Schur complement systems of highly-indefinite linear systems for a parallel hybrid solver. Numer. Math., Theory Methods Appl. 3, 352-366 (2010)

10. Puntanen, S, Styan, PH: Schur complements in statistics and probability. In: The Schur Complement and Its Applications. Numerical Methods and Algorithms, vol. 4, pp. 163-226 (2005)

11. Ouellette, DV: Schur complements and statistics. Linear Algebra Appl. 36, 187-295 (1981)

12. Zhang, FZ: The Schur Complement and Its Applications. Springer, New York (2005)

13. Kolotilina, LY: Nonsingularity/singularity criteria for nonstrictly block diagonally dominant matrices. Linear Algebra Appl. 359, 133-159 (2003)

14. You, ZY, Jiang, ZQ: The diagonal dominance of block matrices. J. Xi'an Jiaotong Univ. 18, 123-125 (1984)

15. Robert, F: Block H-matrices et convergence des methods iterations classiques par blocs. Linear Algebra Appl. 2, 223-265 (1969)

16. Polman, B: Incomplete blockwise factorizations of (block) H-matrices. Linear Algebra Appl. 90, 119-132 (1987)

17. Zhang, CY, Li, YT, Chen, F: On Schur complement of block diagonally dominant matrices. Linear Algebra Appl. 414, 533-546 (2006)

18. Feingold, DG, Varga, RS: Block diagonally dominant matrices and generalizations of the Gerschgorin circle theorem. Pac. J. Math. 12, 1241-1250 (1962)

19. Saad, Y, Schultz, MH: GMRES: a generalized minimal residual algorithm for solving nonsymmetric linear systems. SIAM J. Sci. Stat. Comput. 7, 856-869 (1986) 